\title{
Synthesis, Antimalarial Activity Evaluation and Drug- likeness Study of Some New Quinoline-Lawsone Hybrids
}

\author{
A. KASHYAP, D. CHETIAAND M. RUDRAPAL*
}

Department of Pharmaceutical Sciences, Dibrugarh University, Dibrugarh-786 001, India

Kashyap, et al.: Antimalarial Activity of Quinoline-Lawsone Hybrids

In the present study, a new series of quinoline-lawsone hybrid compounds were synthesized and evaluated in vitro for their antimalarial effectiveness. Several aliphatic and aromatic/heteroaromatic diamines were used as connecting/bridge moiety between the 7-chloro-4-aminoquinoline and 3-amino-1, 4-naphthoquinone pharmacophoric scaffolds. The molecular properties of hybrid compounds were also studied in silico for drug-likeness assessment based on Lipinski's rule of five. Results of antimalarial activity reveal that all the tested compounds showed activity against both chloroquine sensitive (RKL-2) and chloroquine resistant (RKL-9) strains of Plasmodium falciparum which was considerably less as compared to the standard drug, chloroquine. All the compounds exhibited same degree of activity at the tested dose against sensitive strain with $\mathrm{IC}_{50}$ values $0.391-1.033 \mu \mathrm{g} / \mathrm{ml}$. Furthermore, four compounds which were additionally tested against resistant strain also showed activity with $\mathrm{IC}_{50}$ values from 0.684 to $1.778 \mu \mathrm{g} / \mathrm{ml}$ at the same dose. The $\mathrm{IC}_{50}$ values for CQ against sensitive and resistant strains of $P$. falciparum were found to be $0.0391 \mu \mathrm{g} / \mathrm{ml}$ and $0.305 \mu \mathrm{g} / \mathrm{ml}$, respectively. From results it is apparent that the compound with a small alkyl bridge moiety diaminoethyl possesses better activity profile against both sensitive and resistant $\mathbf{s t r a i n s}_{\left(\mathrm{IC}_{50}=0.391 \text { and }\right.}$ $0.684 \mu \mathrm{g} / \mathrm{ml}$, respectively) than rest of the synthesized analogues. The results of drug-likeness studies showed that all newly designed quinoline-lawsone hybrids possess good drug-like properties, indicating their druglikeness behaviour is favourable for optimal antimalarial action. Assessment of drug-likeness score further implies the suitability of hybrid derivatives as drug-like molecules.

Key words: Quinoline, lawsone, hybrid, bridge moiety, Plasmodium falciparum, resistant malaria

Over the last few decades, the burden of malaria has increasingly become a serious health concern around the globe. The disease remains one of the most lethal infectious diseases of human beings affecting around 300-600 million people with about three million deaths per year globally ${ }^{[1]}$. Plasmodium falciparum is the most prevalent and deadly species among all malaria parasites, which causes severe complicated malaria such as cerebral malaria, and is responsible for most of the malaria-related deaths worldwide. Recent emergence of multidrug-resistant strains of $P$. falciparum has further complicated the issues of treating malaria using currently available antimalarial $\operatorname{drugs}^{[2-4]}$. However, with the development of novel and potent antimalarial agents the above challenging issue could be addressed.

Chloroquine (CQ, fig. 1), a 4-aminoquinoline synthetic antimalarial, was once a highly potent drug against malaria. Literature reports suggest that 7-chloro4-aminoquinoline nucleus of 4-aminoquinoline

*Address for correspondence E-mail: rudrapal.m03@gmail.com

November-December 2016 antimalarial drugs is responsible for their antimalarial action which could be attributed mainly due to the prevention of toxic haem polymerization into nontoxic hemozoin (malaria pigment) by inhibiting parasitic enzyme, particularly heme polymerase, which ultimately leads to death of parasites ${ }^{[2,5]}$. However, $P$. falciparum resistant to $\mathrm{CQ}$ has seriously impaired the use of this important drug and other synthetic quinoline antimalarials such as mefloquine in the treatment of malaria ${ }^{[6]}$. Novel artemisinin-based antimalarials (artemether and artesunate) and their combination therapies such as mefloquine-artesunate which are currently used for treating resistant malaria have also become clinically less effective against

This is an open access article distributed under the terms of the Creative Commons Attribution-NonCommercial-ShareAlike 3.0 License, which allows others to remix, tweak, and build upon the work non-commercially, as long as the author is credited and the new creations are licensed under the identical terms

Accepted 27 November 2016

Revised 19 October 2016

Received 10 June 2016

Indian J Pharm Sci 2016;78(6):801-809 
multidrug resistant $P$. falciparum malaria ${ }^{[7,8]}$. On the other hand, atovaquone (ATQ, fig. 1), a derivative of lawsone (2-hydroxy-1,4-naphthoquinone) is an useful antimalarial agent, but it has less clinical efficacy when used alone. ATQ is used in combination with proguanil for the prophylaxis of $P$. falciparum malaria. The antiparasitic activity of the quinones is exhibited by several mechanisms such as competitive inhibition of the cytochrome bc1 complex, generation of reactive oxygen species (ROS), enzymatic inhibition (glutathione reductase), and alkylation ${ }^{[9,10]}$.

To combat the problem of drug resistance, combination therapy has been used for years with limited success, and therefore, the concept of molecular hybridization has recently been introduced for the development of new drugs for treating multidrug resistant $P$. falciparum malaria. The design of hybrid molecules therefore remains to be an effective alternative approach in rational drug design paradigm, which involves covalent fusion of two or more pharmacophore units into a single molecular entity with distinct pharmacodynamic property targeting multiple therapeutic targets ${ }^{[3,11,12]}$. A single hybrid molecule therefore exhibit improved therapeutic response just similar to synergistic like drug action. Further, desired antimalarial efficacy could be achieved with resistant preventing action. This strategy not only remains therapeutically efficacious but also cost effective which in turn reduces overall drug pressure of the treatment. Some hybrid molecules like 4-aminoquinoline-trioxane, CQ-ferrocene, and artemisinin-mefloquine hybrids are under clinical trials as potent antimalarial agents ${ }^{[13-16]}$.

In view of above facts, an attempt was made to design some novel antimalarial derivatives through coupling between the structural scaffolds of quinoline (7-chloro4-aminoquinoline, a residue from CQ) and lawsone (2-hydroxy-1,4-naphthoquinone, a residue from ATQ)

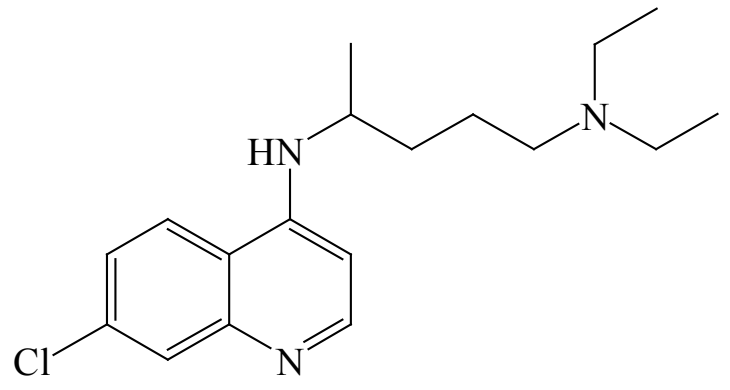

CQ based on molecular hybridization strategy (fig. 2). The hybrid molecules are conjugates of quinoline-lawsone with an alkyl or aryl group which acts as the bridge (connecting) moiety. Newer hybrid molecules would be potent with activity against resistant strains of $P$. falciparum malaria.

In the present study, some new hybrid molecules of quinoline (4-amino(substituted)-7-chloroquinoline) and lawsone (2-hydroxy-1,4-naphthoquinone) were designed and synthesized with an aim to evaluate their antimalarial activity. The synthesized compounds were characterised for structural determination by physiochemical and spectroscopic methods. The molecular properties of compounds were also studied in silico for drug-likeness (DL) assessment based on Lipinski's rule of five with some additional parameters.

\section{MATERIALS AND METHODS}

All chemicals used were procured commercially from Sigma Aldrich Corporation (USA), Merck Specialists Pvt. Ltd. (Germany), HiMedia Lab. Pvt. Ltd. (Germany) or Spectrochem Pvt. Ltd. (India), and were used without further purification. The solvents and reagents used in the antimalarial study were of analytical grade. The progress of reactions was monitored by the silica gel-G thin layer chromatography (TLC) and the spots were visualized by iodine vapours. Melting points (MP) were measured in open capillaries on an electrically heated melting point apparatus. UV/Vis spectra were recorded on Shimadzu UV-1700 UV/Vis spectrophotometer and the wavelength of maximum absorption $\left(\lambda_{\text {max }}, \mathrm{nm}\right)$ is reported. Infrared (IR) spectra were obtained on a Bruker Alpha Fourier transform infrared (FTIR) spectrometer by attenuated total reflection (ATR) technique and are reported in terms of frequency of absorption $\left(\mathrm{v}, \mathrm{cm}^{-1}\right) .{ }^{1} \mathrm{H}$ and ${ }^{13} \mathrm{C}$ nuclear magnetic resonance (NMR) spectra were recorded on

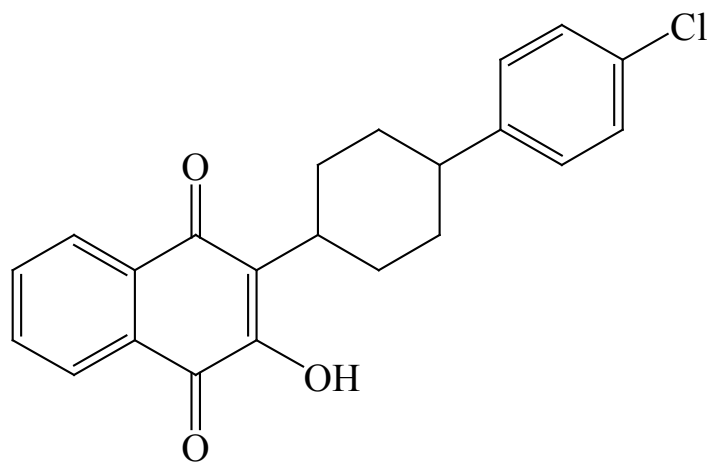

ATQ

Fig. 1: Structures of chloroquine (CQ) and atovaquone (ATQ). 
<smiles>CC1=C(O)C(=O)c2ccccc2C1=O</smiles>

Lawsone residue (ATQ)<smiles>Nc1ccnc2cc(Cl)ccc12</smiles>

Quinoline residue (CQ)

Molecular hybridization<smiles>[X][NH2+]NCNC</smiles>

Lawsone-Quinoline hybrid

Fig. 2: Design of quinoline-lawsone hybrids.

Bruker AD II 400 FT-NMR spectrometer at 400 and 100 $\mathrm{MHz}$, respectively using tetramethylsilane (TMS) as an internal standard $(\delta 0.00 \mathrm{ppm})$ and $\mathrm{D}_{2} \mathrm{O}$ (deuterated water) as a solvent. Chemical shift $(\delta)$ values were expressed in parts per million (ppm) relative to TMS $(\delta 0.00 \mathrm{ppm})$.

\section{General procedure of synthesis:}

The chlorination of lawsone, 1 with thionyl chloride yielded 3-chloro derivative of lawsone, i.e., 3-chloro2-hydroxy-1,4-naphthoquinone, 2, which was later reacted with different $\mathrm{N}$-substituted 7-chloro4-aminoquinolines, 4 a-f to obtain target hybrid compounds, 5a-f. $N$-substituted quinolines were previously obtained from the reaction between commercially available 4,7-dichloroqunoline, 3 with different aliphatic/aromatic diamines. The general scheme of synthesis of target compounds is depicted in fig. 3.

To a mixture of 2-hydroxy-1,4-naphthoquinone ( $7 \mathrm{~g}$, $40 \mathrm{~m} / \mathrm{mol})$ and pyridine $(3.2 \mathrm{~g}, 3.2 \mathrm{ml}, 40 \mathrm{~m} / \mathrm{mol})$, thionyl chloride $(6 \mathrm{ml})$ was added drop wise, and the resulting solution was heated under reflux at $70^{\circ}$ for

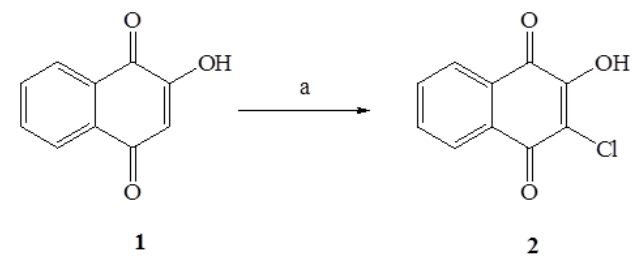

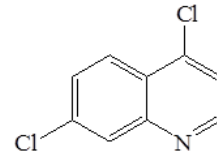
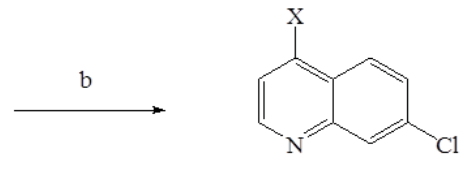

4a-f

2, c

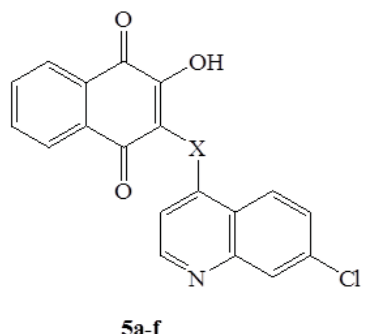

Fig. 3: Synthetic scheme of target hybrid compounds (5a-f). Reagents and conditions: (a) $\mathrm{SOCl}_{2}$, pyridine, $70^{\circ}, 1 \mathrm{~h}$; (b) aliphatic/aromatic diamine, $80^{\circ}$ for $1 \mathrm{~h}, 120-130^{\circ}$ for $8 \mathrm{~h}$; (c) EtOH, reflux, $12 \mathrm{~h}$, hybrid molecules, 5a-f, 5a:X=-NH$\left(\mathrm{CH}_{2}\right)_{2}-\mathrm{NH} ; 5 \mathrm{~b}: \mathrm{X}=-\mathrm{NH}-\left(\mathrm{CH}_{2}\right)_{3}-\mathrm{NH}-;$ 5c: $\mathrm{X}=-\mathrm{NH}-\mathrm{C}_{6} \mathrm{H}_{4}-\mathrm{NH}-;$ 5d: $\mathrm{X}=-\mathrm{NH}-\mathrm{CH}_{2}-\mathrm{CH}\left(\mathrm{C}_{10} \mathrm{H}_{7}\right)-\mathrm{NH}-;$ 5e: $\mathrm{X}=-\left(\mathrm{N}\left(\mathrm{CH}_{2}\right)_{2} ; 5 \mathrm{f}: \mathrm{X}=-\mathrm{NH}-\right.$ $\left(\mathrm{C}_{6} \mathrm{H}_{3} \mathrm{~N}\right)-\mathrm{NH}-$

about $1 \mathrm{~h}$. The solvent was removed under vacuum and the product thereby obtained was separated ${ }^{[11]}$.

A mixture of 4,7-dichloroquinoline $(5 \mathrm{~g}, 25.18 \mathrm{~m} / \mathrm{mol})$ and respective aliphatic/aromatic diamine $(50.36 \mathrm{~m} /$ mol) was heated slowly from room temperature to $80^{\circ}$ over $1 \mathrm{~h}$ with stirring and subsequently at $120-130^{\circ}$ for $8 \mathrm{~h}$ with continued stirring to drive the reaction to completion. Then, the reaction mixture was cooled to room temperature and transferred to dichloromethane $(80 \mathrm{ml})$. The resulting mixture was washed with sodium hydroxide $(1 \mathrm{~mol}, 80 \mathrm{ml})$ and brine $(80 \mathrm{ml})$ to give an aqueous layer and an organic layer containing the desired product. The organic layer was dried over anhydrous sodium sulfate, and the solvent was removed under reduced pressure to yield the product as a yellowish white solid. The crude solid was recrystallized from dichloromethane to get the pure compound ${ }^{[2]}$.

To a solution of $N$-substituted 7-chloro-4aminoquinolines $(17 \mathrm{~m} / \mathrm{mol})$ in ethanol $(20 \mathrm{ml})$, 3-chloro-2-hydroxy-1,4-naphthoquinone (3.8 g, 18 
$\mathrm{m} / \mathrm{mol}$ ) was added and the mixture was heated under reflux for about $12 \mathrm{~h}$. The solution was concentrated under reduced pressure to give a viscous mass which was subsequently poured into an ice cold ammonium hydroxide. The sticky solid thus separated was dissolved in dichloromethane and was extracted with alkaline solution. The organic solution was washed with water, dried over anhydrous sodium sulphate and then evaporated to dryness under reduced pressure to yield the crude product. The product was recrystallized from methano[ ${ }^{[11,17]}$.

\section{In vitro antimalarial activity evaluation:}

All the synthesized compounds, 5a-f were screened for in vitro antimalarial activity against a CQ-sensitive strain of $P$. falciparum (RKL-2, Rourkela, Odisha, India) and additionally, four compounds, 5a, 5d-f were also screened for the activity against CQ-resistant strain of P. falciparum (RKL-9, Rourkela, Odisha, India). Four compounds were selected for the activity against resistant strain (RKL-9) based on their superior activity profile on sensitive strain (RKL-2) than rest of the analogues in the series. The antimalarial activity screening was carried out by Giemsa stained slide method as follows:

The laboratory adapted strain of $P$. falciparum was routinely cultured in vitro at $37^{\circ}$ temperature and $5 \%$ $\mathrm{CO}_{2}$ environment in RPMI (Roswell Park Memorial Institute)-1640 medium supplemented with $25 \mathrm{~m} / \mathrm{M}$ HEPES [(N-2-hydroxyethylpiperazine-N-2-ethane sulfonic acid)], 1\% D-glucose, $0.23 \%$ sodium bicarbonate, gentamycin $(40 \mathrm{mg} / \mathrm{ml})$, amphotericin-B $(0.25 \mathrm{mg} / \mathrm{ml})$ and $10 \%$ heat inactivated human serum. For antimalarial testing, the asynchronous parasites of $P$. falciparum were synchronized by $5 \%$ D-sorbitol treatment to obtain only the ring stage parasitized cells. For carrying out the assay, the initial ring stage parasitaemia of $0.8-1.5 \%$ in $3 \%$ haematocrit in a total volume of $100 \mathrm{ml}$ using RPMI-1640 medium was uniformly maintained. A stock solution $(1 \mathrm{mg} / \mathrm{ml})$ of the test compound was prepared in dimethyl sulfoxide (DMSO) and subsequent dilutions were made with the incomplete RPMI culture medium. A total of 100 $\mu 1$ of the test compound at $0.1 \mathrm{mg} / \mathrm{ml}$ concentration (in duplicate) was incubated with parasitized cell preparation at $37^{\circ}$ and $5 \% \mathrm{CO}_{2}$ in a $\mathrm{CO}_{2}$ incubator. After an incubation period of 36-40 h, blood smears were prepared from each well and stained with $3 \%$ Giemsa stain. The slides were observed under the light microscope and the percentage dead rings + trophozoites were calculated against 200 asexual parasites with respect to the control group. $\mathrm{CQ}(0.1 \mathrm{mg} / \mathrm{ml})$ was used as the standard reference drug. Test results were compared with the standard result of CQ. Each test compound was assayed in two replicates and counted against 200 asexual parasites (\% dead rings+trophozoites) per replicate. The mean of percentage of inhibition of parasite growth was calculated for duplicate studies. Further, the minimum inhibitory concentration (MIC) and inhibitory concentration at $50 \%$ of maximal inhibition $\left(\mathrm{IC}_{50}\right)$ values (in $\mu \mathrm{g} / \mathrm{ml}$ ) were also calculated using the NonLin v1.1 software ${ }^{[11,18-20]}$.

\section{DL prediction:}

The DL properties of the synthesized compounds, 5a-f were studied in silico using web-based Molsoft (molsoft.com/molprop/) and Molinspiration Cheminformatics (www.molinspiration.com) software. The following molecular descriptors were calculated for DL prediction: molecular weight (MW), water solubility (LogS), octanol-water partition coefficient (LogP), hydrogen bond acceptor (HBA) and hydrogen bond donor (HBD) count, polar surface area (PSA), molar volume (MV) and rotable bonds. The DL score were also calculated for all the studied compounds.

\section{RESULTS AND DISCUSSION}

In the present study, a new series of lawsone-quinolinelawsone hybrid compounds were synthesized and evaluated in vitro for antimalarial activity. Several aliphatic and aromatic/heteroaromatic diamines were used as conjugating moiety between quinoline (7-chloro-4-aminoquinoline, a residue of CQ) and lawsone (2-hydroxy-1,4-naphthoquinone, a residue of ATQ) pharmacophores. The purity of the synthesized compounds was ascertained by melting point determinations and silica gel G TLC. All the compounds were obtained in good yields with high purity. The physicochemical details including MP and $R_{f}$ of the synthesized compounds are presented in Table 1. The structural assignments of the synthesized compounds were made on the basis of UV, FT-IR, ${ }^{1} \mathrm{HNMR}$ and ${ }^{13} \mathrm{CNMR}$ spectral studies. The spectral data of 5a-f are depicted as follows:

2-(2-(7-chloroquinolin-4-ylamino)ethylamino)-3hydroxynaphthalene-1,4-dione (5a): UV spectrum $(\mathrm{MeOH}), \lambda_{\max }(\mathrm{nm}): 305.5$; IR (ATR), $v\left(\mathrm{~cm}^{-1}\right): 3668.73$ $(\mathrm{O}-\mathrm{H}), 3360.36,3338.02(2 \mathrm{~N}-\mathrm{H}), 3025.32,3004.89$ (C-H, aryl), 2865.35, $2834.60\left(\mathrm{C}-\mathrm{H}, \mathrm{CH}_{2}\right), 1678.48$, $1658.20(2 \mathrm{C}=\mathrm{O}), 1372.20,1268.60(\mathrm{C}-\mathrm{N}), 1049.59$ 
TABLE 1: PHYSICOCHEMICAL DATA

\begin{tabular}{lllllccc}
\hline Compound code Molecular formula & Molecular weight & state & Colour & Yield (\%) & Melting point $\left(^{\circ}\right)$ & $R_{\mathrm{f}}$ value \\
\hline $5 \mathrm{a}$ & $\mathrm{C}_{21} \mathrm{H}_{16} \mathrm{~N}_{3} \mathrm{O}_{3} \mathrm{Cl}$ & 393.83 & Solid & Brown & 84.44 & $150-152$ & 0.77 \\
$5 \mathrm{~b}$ & $\mathrm{C}_{22} \mathrm{H}_{18} \mathrm{~N}_{3} \mathrm{O}_{3} \mathrm{Cl}$ & 407.86 & Solid & Orange & 66.76 & $256-261$ & 0.51 \\
$5 \mathrm{c}$ & $\mathrm{C}_{25} \mathrm{H}_{16} \mathrm{~N}_{3} \mathrm{O}_{3} \mathrm{Cl}$ & 441.87 & Solid & Brown & 71.05 & $194-199$ & 0.57 \\
$5 \mathrm{~d}$ & $\mathrm{C}_{31} \mathrm{H}_{22} \mathrm{~N}_{3} \mathrm{O}_{3} \mathrm{Cl}$ & 519.99 & Solid & Brown & 52.96 & $306-309$ & 0.55 \\
$5 \mathrm{e}$ & $\mathrm{C}_{23} \mathrm{H}_{18} \mathrm{~N}_{3} \mathrm{O}_{3} \mathrm{Cl}$ & 419.87 & Solid & Brown & 67.33 & $175-177$ & 0.65 \\
$5 \mathrm{f}$ & $\mathrm{C}_{24} \mathrm{H}_{15} \mathrm{~N}_{4} \mathrm{O}_{3} \mathrm{Cl}$ & 442.08 & Solid & Brown & 59.22 & $171-173$ & 0.69 \\
\hline
\end{tabular}

*Solvent system, acetone/MeOH-1:2

(C-Cl, aryl); ${ }^{1} \mathrm{H}$ NMR (400 MHz, $\left.\mathrm{D}_{2} \mathrm{O}\right), \delta$ (ppm): 9.65 (bs, $1 \mathrm{H}, \mathrm{OH}), 8.20\left(\mathrm{~d}, 1 \mathrm{H}, \mathrm{J}=4.3 \mathrm{~Hz}\right.$, quinoline $\left.-\mathrm{H}_{8}\right)$, $8.00\left(\mathrm{~d}, 1 \mathrm{H}, \mathrm{J}=6.8 \mathrm{~Hz}\right.$, quinoline- $\left.\mathrm{H}_{5}\right), 7.70(\mathrm{dd} 1 \mathrm{H}$, $\mathrm{J}=4.6,4.5 \mathrm{~Hz}$, quinoline- $\left.\mathrm{H}_{6}\right), 7.40(\mathrm{~d}, 1 \mathrm{H}, \mathrm{J}=7.5 \mathrm{~Hz}$, quinoline- $\left.\mathrm{H}_{2}\right), 7.30\left(\mathrm{~d}, 1 \mathrm{H}, \mathrm{J}=8.2 \mathrm{~Hz}\right.$, quinoline- $\left.\mathrm{H}_{3}\right)$, $6.80(\mathrm{~m}, 4 \mathrm{H}, \mathrm{Ar}-\mathrm{H}), 5.24(\mathrm{~s}, 1 \mathrm{H}, \mathrm{NH}), 4.34(\mathrm{~s}, 1 \mathrm{H}, \mathrm{NH})$, 3.26-3.17 (t, $2 \mathrm{H}, \mathrm{J}=8.4 \mathrm{~Hz}, \mathrm{NH}-\mathrm{CH}_{2} \mathrm{CH}_{2}-\mathrm{NH}$ ), 2.56$2.21\left(\mathrm{t}, 2 \mathrm{H}, \mathrm{J}=7.6 \mathrm{~Hz}, \mathrm{NH}-\mathrm{CH}_{2} \mathrm{CH}_{2}-\mathrm{NH}\right) ;{ }^{13} \mathrm{C} \mathrm{NMR}$ $\left(100 \mathrm{MHz}, \mathrm{D}_{2} \mathrm{O}\right), \delta(\mathrm{ppm}): 178.32,172.02(2 \mathrm{C}=\mathrm{O})$, 154.24, (C-OH), 150.94 (C-2, quinoline), 148.34 (C8a, quinoline), 138.42 (C-7, quinoline), 136.28 (C8, quinoline), 130.63 (C-6, quinoline), 128.07 (C-5, quinoline), 126.48, 124.86, 120.32, 118.05 (aromatic C), 116.36 (C-4a, quinoline), 115.02 (C-2, quinoline), 120.13 (olefinic C), 45.28, $28.09\left(\mathrm{CH}_{2}\right)$.

2-(3-(7-chloroquinolin-4-ylamino)propylamino)-3hydroxynaphthalene-1,4-dione (5b): UV spectrum $(\mathrm{MeOH}), \lambda_{\text {max }}(\mathrm{nm}): 234.0$; IR (ATR), $v\left(\mathrm{~cm}^{-1}\right): 3544.08$ $(\mathrm{O}-\mathrm{H}), 3350.24,3328.56$ (2 N-H), 3128.05, 3030.45, 3015.26 (C-H, aryl), 1678.48, 1665.23 (2 C=O), 1298.31, $1265.29(\mathrm{C}-\mathrm{N}), 1078.13\left(\mathrm{C}-\mathrm{Cl}\right.$, aryl); ${ }^{1} \mathrm{H}$ NMR (400 MHz, $\left.\mathrm{D}_{2} \mathrm{O}\right), \delta(\mathrm{ppm}): 8.46$ (bs, 1H, OH), $8.20\left(\mathrm{~d}, 1 \mathrm{H}, \mathrm{J}=4.8 \mathrm{~Hz}\right.$, quinoline $\left.-\mathrm{H}_{8}\right), 8.00(\mathrm{~d}, 1 \mathrm{H}$, $\mathrm{J}=6.6 \mathrm{~Hz}$, quinoline $\left.-\mathrm{H}_{5}\right), 7.56(\mathrm{dd} 1 \mathrm{H}, \mathrm{J}=4.8,4.6 \mathrm{~Hz}$, quinoline $\left.-\mathrm{H}_{6}\right), 7.38\left(\mathrm{~d}, 1 \mathrm{H}, \mathrm{J}=7.4 \mathrm{~Hz}\right.$, quinoline $\left.-\mathrm{H}_{2}\right)$, $7.28\left(\mathrm{~d}, 1 \mathrm{H}, \mathrm{J}=8.4 \mathrm{~Hz}\right.$, quinoline- $\left.\mathrm{H}_{3}\right), 6.28(\mathrm{~m}, 4 \mathrm{H}$, Ar-H), 5.35 (s, 1H, NH), $4.36(\mathrm{~s}, 1 \mathrm{H}, \mathrm{NH}), 3.28-$ $3.10\left(\mathrm{t}, 2 \mathrm{H}, \mathrm{J}=8.2 \mathrm{~Hz}, \mathrm{NH}-\mathrm{CH}_{2} \mathrm{CH}_{2}-\mathrm{NH}\right), 2.58-$ $2.22\left(\mathrm{t}, 2 \mathrm{H}, \mathrm{J}=7.4 \mathrm{~Hz}, \mathrm{NH}-\mathrm{CH}_{2} \mathrm{CH}_{2}-\mathrm{NH}\right) ;{ }^{13} \mathrm{C} \mathrm{NMR}$ (100 MHz, $\left.\mathrm{D}_{2} \mathrm{O}\right), \delta(\mathrm{ppm}): 178.20,172.00(2 \mathrm{C}=\mathrm{O})$, 154.65, (C-OH), 152.26 (C-2, quinoline), 148.26 (C8a, quinoline), 140.36 (C-7, quinoline), 136.24 (C8, quinoline), 132.24 (C-6, quinoline), 128.00 (C-5, quinoline), 126.48, 124.04, 120.78, 118.60 (aromatic C), 116.46 (C-4a, quinoline), 115.24 (C-2, quinoline), 120.43 (olefinic C), 45.48, $28.42\left(\mathrm{CH}_{2}\right)$.

2-(2-(7-chloroquinolin-4-ylamino)phenylamino)-3hydroxynaphthalene-1,4-dione (5c): UV spectrum $(\mathrm{MeOH}), \lambda_{\text {max }}(\mathrm{nm}): 331.3$; IR (ATR), $v\left(\mathrm{~cm}^{-1}\right): 3542.29$ (O-H), 3328.21, 3316.20 (2 N-H), 3120.02, 3030.26
(C-H， aryl)，2960.26，2942.02，2840.28，2834.20 $\left(\mathrm{C}-\mathrm{H}, \mathrm{CH}_{3}\right.$ and $\left.\mathrm{CH}_{2}\right), 1678.24,1656.49(2 \mathrm{C}=\mathrm{O})$, 1288.45, 1278.24 (C-N), 1065.24 (C-Cl, aryl); ${ }^{1} \mathrm{H}$ NMR (400 MHz, D 2 ), $\delta(\mathrm{ppm}): 8.45(\mathrm{bs}, 1 \mathrm{H}, \mathrm{OH})$, $8.20\left(\mathrm{~d}, 1 \mathrm{H}, \mathrm{J}=8.6 \mathrm{~Hz}\right.$, quinoline- $\left.\mathrm{H}_{8}\right), 7.86(\mathrm{~d}, 1 \mathrm{H}$, $\mathrm{J}=6.8 \mathrm{~Hz}$, quinoline- $\left.\mathrm{H}_{5}\right), 7.58(\mathrm{dd} 1 \mathrm{H}, \mathrm{J}=4.8,4.8 \mathrm{~Hz}$, quinoline- $\left.\mathrm{H}_{6}\right), 7.39\left(\mathrm{~d}, 1 \mathrm{H}, \mathrm{J}=7.6 \mathrm{~Hz}\right.$, quinoline- $\left.\mathrm{H}_{2}\right)$, $7.24\left(\mathrm{~d}, 1 \mathrm{H}, \mathrm{J}=8.8 \mathrm{~Hz}\right.$, quinoline- $\left.\mathrm{H}_{3}\right), 6.58(\mathrm{~m}, 4 \mathrm{H}$, Ar-H), 6.26 (m, 4H, Ar-H), 5.44 (s, 1H, NH), 4.64 $(\mathrm{s}, 1 \mathrm{H}, \mathrm{NH}) ;{ }^{13} \mathrm{C} \mathrm{NMR}\left(100 \mathrm{MHz}, \mathrm{D}_{2} \mathrm{O}\right), \delta(\mathrm{ppm})$ : 176.00, $172.48(2 \mathrm{C}=\mathrm{O}), 156.40,(\mathrm{C}-\mathrm{OH}), 152.40(\mathrm{C}-$ 2, quinoline), 148.24 (C-8a, quinoline), 142.51 (C7, quinoline), 136.63 (C-8, quinoline), 137.22 (C-6, quinoline), 128.90 (C-5, quinoline), 126.06, 124.50, 122.24, 120.68, 118.42 (aromatic C), 116.60 (C-4a, quinoline), 115.52 (C-2, quinoline), 120.46 (olefinic C).

2-(2-(7-chloroquinolin-4-ylamino)-1-(naphthalen-1yl)ethylamino)-3-hydroxynaphthalene-1,4-dione (5d): UV spectrum $(\mathrm{MeOH}), \lambda_{\text {max }}(\mathrm{nm}): 270.0$; IR (ATR), v $\left(\mathrm{cm}^{-1}\right): 3498.24(\mathrm{O}-\mathrm{H}), 3345.68,3328.12(2 \mathrm{~N}-\mathrm{H})$, 3128.24, 3068.34 (C-H, aryl), 2960.26, 2942.02, 2846.12, $2824.56\left(\mathrm{C}-\mathrm{H}, \mathrm{CH}\right.$ and $\left.\mathrm{CH}_{2}\right), 1666.46$, $1646.28(2 \mathrm{C}=\mathrm{O}), 1278.20,1264.20(\mathrm{C}-\mathrm{N}), 1064.76$ (C-Cl, aryl); ${ }^{1} \mathrm{H}$ NMR (400 MHz, $\left.\mathrm{D}_{2} \mathrm{O}\right), \delta$ (ppm): 8.44 (bs, $1 \mathrm{H}, \mathrm{OH}), 8.26\left(\mathrm{~d}, 1 \mathrm{H}, \mathrm{J}=8.6 \mathrm{~Hz}\right.$, quinoline- $\left.\mathrm{H}_{8}\right)$, $8.05\left(\mathrm{~d}, 1 \mathrm{H}, \mathrm{J}=6.4 \mathrm{~Hz}\right.$, quinoline- $\left.\mathrm{H}_{5}\right), 7.58(\mathrm{dd} 1 \mathrm{H}$, $\mathrm{J}=4.6,4.4 \mathrm{~Hz}$, quinoline- $\left.\mathrm{H}_{6}\right), 7.42(\mathrm{~d}, 1 \mathrm{H}, \mathrm{J}=7.4 \mathrm{~Hz}$, quinoline- $\left.\mathrm{H}_{2}\right), 7.22\left(\mathrm{~d}, 1 \mathrm{H}, \mathrm{J}=8.4 \mathrm{~Hz}\right.$, quinoline- $\left.\mathrm{H}_{3}\right)$, $6.64(\mathrm{~m}, 4 \mathrm{H}, \mathrm{Ar}-\mathrm{H}), 6.28(\mathrm{~m}, 4 \mathrm{H}, \mathrm{Ar}-\mathrm{H}), 5.40(\mathrm{~s}, 1 \mathrm{H}$, $\mathrm{NH}), 4.36(\mathrm{~s}, 1 \mathrm{H}, \mathrm{NH}), 3.20-3.11(\mathrm{t}, 2 \mathrm{H}, \mathrm{J}=8.6 \mathrm{~Hz}$, $\left.\mathrm{NH}_{-} \mathrm{CHCH}_{2}-\mathrm{NH}\right), 2.68-2.42(\mathrm{t}, 2 \mathrm{H}, \mathrm{J}=6.8 \mathrm{~Hz}, \mathrm{NH}-$ $\left.\mathrm{CHCH}_{2}-\mathrm{NH}\right) ;{ }^{13} \mathrm{C}$ NMR $\left(100 \mathrm{MHz}, \mathrm{D}_{2} \mathrm{O}\right), \delta$ (ppm): 176.84, $174.26(2 \mathrm{C}=\mathrm{O}), 158.24,(\mathrm{C}-\mathrm{OH}), 152.60(\mathrm{C}-$ 2, quinoline), 148.18 (C-8a, quinoline), 142.49 (C7, quinoline), 136.37 (C-8, quinoline), 136.44 (C-6, quinoline), 128.12 (C-5, quinoline), 126.26, 124.00, 122.212 120.19, 118.06 (aromatic C), 116.44 (C-4a, quinoline), 115.27 (C-2, quinoline), 120.44 (olefinic C), 48.54, $29.04\left(\mathrm{CH}, \mathrm{CH}_{2}\right)$.

2-(4-(7-chloroquinoline-4-yl)piperazin-1-yl)-3- 
hydroxynapthalene-1,4-dione (5e): UV spectrum $(\mathrm{MeOH}), \quad \lambda_{\max }(\mathrm{nm}): 236.0$; IR (ATR), $v\left(\mathrm{~cm}^{-1}\right)$ : $3528.04(\mathrm{O}-\mathrm{H}), 3360.36,3028.45,3018.03(\mathrm{C}-\mathrm{H}$, aryl), 2874.09, $2848.46\left(\mathrm{C}-\mathrm{H}, \mathrm{CH}_{2}\right), 1678.00,1629.42$ (2 $\mathrm{C}=\mathrm{O}), 1378.04,1258.62(\mathrm{C}-\mathrm{N}), 1048.05(\mathrm{C}-\mathrm{Cl}$, aryl); ${ }^{1} \mathrm{H}$ NMR (400 MHz, $\left.\mathrm{D}_{2} \mathrm{O}\right), \delta(\mathrm{ppm}): 8.25$ (bs, $1 \mathrm{H}$, $\mathrm{OH}), 8.0\left(\mathrm{~d}, 1 \mathrm{H}, \mathrm{J}=8.6 \mathrm{~Hz}\right.$, quinoline $\left.-\mathrm{H}_{8}\right), 7.94(\mathrm{~d}, 1 \mathrm{H}$, $\mathrm{J}=6.6 \mathrm{~Hz}$, quinoline- $\left.\mathrm{H}_{5}\right), 7.60(\mathrm{dd} 1 \mathrm{H}, \mathrm{J}=4.8,4.8 \mathrm{~Hz}$, quinoline- $\left.\mathrm{H}_{6}\right), 7.40\left(\mathrm{~d}, 1 \mathrm{H}, \mathrm{J}=7.8 \mathrm{~Hz}\right.$, quinoline $\left.-\mathrm{H}_{2}\right)$, $7.36\left(\mathrm{~d}, 1 \mathrm{H}, \mathrm{J}=8.4 \mathrm{~Hz}\right.$, quinoline- $\left.\mathrm{H}_{3}\right), 6.62(\mathrm{~m}, 4 \mathrm{H}, \mathrm{Ar}-$ $\mathrm{H}), 3.26\left(\mathrm{t}, 2 \mathrm{H}, \mathrm{J}=8.2 \mathrm{~Hz}, \mathrm{~N}-\mathrm{CH}_{2} \mathrm{CH}_{2}-\mathrm{N}\right), 2.42(\mathrm{t}, 2 \mathrm{H}$, $\left.\mathrm{J}=7.8 \mathrm{~Hz}, \mathrm{~N}-\mathrm{CH}_{2} \mathrm{CH}_{2}-\mathrm{N}\right) ;{ }^{13} \mathrm{C} \mathrm{NMR}\left(100 \mathrm{MHz}, \mathrm{D}_{2} \mathrm{O}\right)$, $\delta$ (ppm): 178.50, $172.00(2 \mathrm{C}=\mathrm{O}), 154.04,(\mathrm{C}-\mathrm{OH})$, 152.06 (C-2, quinoline), 148.20 (C-8a, quinoline), 138.44 (C-7, quinoline), 136.42 (C-8, quinoline), 130.58 (C-6, quinoline), 128.64 (C-5, quinoline), 126.80, 124.00, 120.42, 118.04 (aromatic C), 116.75 (C-4a, quinoline), 115.03 (C-2, quinoline), 118.32 (olefinic C), 45.40, $28.08\left(\mathrm{CH}_{2}\right)$.

2-(6-(7-chloroquinolin-4-ylamino)pyridin-2-ylamino)3-hydroxynaphthalene-1,4-dione (5f): UV spectrum $(\mathrm{MeOH}), \lambda_{\max }(\mathrm{nm}): 243.5$; IR (ATR), $v\left(\mathrm{~cm}^{-1}\right): 3424.08$ (O-H), 3340.03, 3328.56 (2 N-H), 3028.40, 3012.26, 2984.12, 2942.67 (C-H, aryl), $1668.12(\mathrm{C}=\mathrm{O})$, 1374.12, $1248.24(\mathrm{C}-\mathrm{N}), 1050.24\left(\mathrm{C}-\mathrm{Cl}\right.$, aryl); ${ }^{1} \mathrm{H}$ NMR (400 MHz, $\left.\mathrm{D}_{2} \mathrm{O}\right), \delta(\mathrm{ppm}): 8.78$ (bs, 1H, OH), $8.24\left(\mathrm{~d}, 1 \mathrm{H}, \mathrm{J}=8.4 \mathrm{~Hz}\right.$, quinoline- $\left.\mathrm{H}_{8}\right), 7.98(\mathrm{~d}, 1 \mathrm{H}$, $\mathrm{J}=6.8 \mathrm{~Hz}$, quinoline- $\left.\mathrm{H}_{5}\right), 7.78(\mathrm{dd} 1 \mathrm{H}, \mathrm{J}=4.8,4.7 \mathrm{~Hz}$, quinoline $\left.-\mathrm{H}_{6}\right), 7.52\left(\mathrm{~d}, 1 \mathrm{H}, \mathrm{J}=7.5 \mathrm{~Hz}\right.$, quinoline $\left.-\mathrm{H}_{2}\right)$, $7.32\left(\mathrm{~d}, 1 \mathrm{H}, \mathrm{J}=6.8 \mathrm{~Hz}\right.$, quinoline- $\left.\mathrm{H}_{3}\right), 6.40(\mathrm{~m}, 4 \mathrm{H}, \mathrm{Ar}-$ $\mathrm{H}), 6.20$ (m, 3H, Ar-H, 2,6-pyridyl), 5.28 (s, 1H, NH), 4.52 (s, 1H, NH); ${ }^{13} \mathrm{C}$ NMR (100 MHz, $\left.\mathrm{D}_{2} \mathrm{O}\right), \delta$ (ppm): 178.42, $172.12(2 \mathrm{C}=\mathrm{O}), 154.10,(\mathrm{C}-\mathrm{OH}), 150.28(\mathrm{C}-$ 2, quinoline), 148.36 (C-8a, quinoline), 138.48 (C7, quinoline), 136.21 (C-8, quinoline), 130.42 (C-6, quinoline), 128.09 (C-5, quinoline), 126.16, 124.22, 120.53, 122.24118 .08 (aromatic C), 116.11 (C-4a, quinoline), 115.43 (C-2, quinoline), 120.13 (olefinic C).

The spectral data depicted above reveal that all the compounds in methanol exhibited characteristic absorption maxima indicating the presence of the chromophore quinoline and naphthoquinone functional moieties. The infrared spectral data showed distinct stretching frequencies for phenolic and alcoholic hydroxyl groups (broad and medium peak for $\mathrm{O}-\mathrm{H}$ stretching), secondary amino groups (narrow and medium peak for $\mathrm{N}-\mathrm{H}$ stretching), cyclic carbonyl groups (sharp and narrow peak $\mathrm{C}=\mathrm{O}$ stretching) and methylene groups (narrow and medium peak for $\mathrm{C}-\mathrm{H}$ stretching), which ascertain the anticipated structure of synthesized compounds. ${ }^{1} \mathrm{H}$ NMR spectra displayed signals for different structural protons of conjugated quinoline-naphthoquinone skeleton where characteristic peak multiplicity pattern was observed for aromatic protons including aromatic components of the bridge moiety. In addition, prominent broad singlet for $\mathrm{OH}$ group, sharp singlet for $\mathrm{NH}$ group and characteristic multiplets for $\mathrm{CH}_{2}$ groups were observed at distinct $\delta$ values for all the synthesized compounds. The ${ }^{13} \mathrm{C}$ resonance data revealed the presence of different carbon atoms of quinoline ring, aryl/heteroaryl system, carbonyl groups and methylene groups present in the structure of synthesized compounds ${ }^{[21]}$.

The in vitro antimalarial activity data is depicted in Table 2. Results are expressed as MIC and $\mathrm{IC}_{50}(\mu \mathrm{g} /$ $\mathrm{ml}$ ) for two independent observations. Results clearly revealed that all the tested compounds showed activity against both CQ-sensitive (RKL-2) and CQ-resistant strains (RKL-9) of P. falciparum which, however, was less than that of the standard reference drug, CQ. All compounds exhibited same degree of activity at the tested dose against sensitive strain. It is clear from results that four compounds, $5 \mathrm{a}, 5 \mathrm{~d}, 5 \mathrm{e}\left(\mathrm{IC}_{50}=0.391 \mu \mathrm{g} /\right.$ $\mathrm{ml})$ and $5 \mathrm{f}\left(\mathrm{IC}_{50}=0.398 \mu \mathrm{g} / \mathrm{ml}\right)$ showed better activity against sensitive strain than rest of the synthesized analogues, $5 \mathrm{~b}\left(\mathrm{IC}_{50}=0.532 \mu \mathrm{g} / \mathrm{ml}\right)$ and $5 \mathrm{c}\left(\mathrm{IC}_{50}=1.033\right.$ $\mu \mathrm{g} / \mathrm{ml})$. The $\mathrm{IC}_{50}$ value for CQ against sensitive strain of P. falciparum was found to be $0.0391 \mu \mathrm{g} / \mathrm{ml}$. Because

\section{TABLE 2: RESULTS OF IN VITRO ANTIMALARIAL ACTIVITY\#}

\begin{tabular}{|c|c|c|c|c|}
\hline \multirow[t]{2}{*}{ Compound code ${ }^{\varsigma}$} & \multicolumn{2}{|c|}{ MIC $(\mu \mathrm{g} / \mathrm{ml})$} & \multicolumn{2}{|c|}{$I C_{50}(\mu \mathrm{g} / \mathrm{ml})$} \\
\hline & RKL-2* & RKL-9** & RKL-2* & RKL-9** \\
\hline $5 a$ & 3.125 & $>25$ & 0.391 & 0.684 \\
\hline $5 b$ & $>25$ & - & 0.532 & - \\
\hline $5 c$ & $>25$ & - & 1.033 & - \\
\hline $5 d$ & 6.25 & $>25$ & 0.391 & 1.171 \\
\hline $5 e$ & 6.25 & $>25$ & 0.391 & 1.091 \\
\hline $5 f$ & 6.25 & $>25$ & 0.398 & 1.778 \\
\hline $\mathrm{CQ}^{\$ S}$ & 6.25 & $>12.5$ & 0.0391 & 0.305 \\
\hline
\end{tabular}

"Data are presented as mean of duplicate observations; *CQ-sensitive $P$. falciparum strain "CQ-resistant $P$. falciparum strain; \$test dose: $5 \mathrm{mg} / \mathrm{ml}$; ${ }^{\$ \varsigma}$ standard dose: $0.1 \mathrm{mg} / \mathrm{ml}$ 
of their superior effectiveness among all compounds in the series these four compounds were additionally screened for activity against resistant strain (RKL-9) of P. falciparum. Compounds $5 \mathrm{a}$ and $5 \mathrm{~d}-5 \mathrm{f}$ showed activity against the resistant strain with $\mathrm{IC}_{50}$ values range from 0.684 to $1.778 \mu \mathrm{g} / \mathrm{ml}$ at the same dose. In this case, the $\mathrm{IC}_{50}$ for CQ was $0.305 \mu \mathrm{g} / \mathrm{ml}$. After careful observation of results, it is apparent that among all synthesized compounds, compound 5a with diaminoethyl bridge moiety showed best activity against both sensitive and resistant strains. Compound 5b (diaminopropyl moiety) and 5c (2, 3-diaminophenyl moiety) were found to be least potent in the series. Rest of the analogues (5d-5f) exhibited good antimalarial activity. These compounds are more active on sensitive strain as compared to the resistant strain of $P$. falciparum. They possess bulky aryl/heteroaryl groups like diamino-1-naphthylethyl, 14-piperidyl and 2,6-diaminopyridyl, respectively as bridge moiety between quinoline and lawsone ring systems. However, the antimalarial activity of all synthesized compounds was found to be comparatively less than that of the standard drug, CQ against both sensitive and resistant strains.

Drug likeness prediction evaluates the acceptability of derivatives as drug molecules based on Lipinski's rule of five ${ }^{[22]}$. In general, drug-like properties are the molecular properties, which include both significant physicochemical descriptors and relevant pharmacokinetic properties. Molecular properties are fundamental structural properties which determine the physicochemical (solubility and permeability) and biochemical (metabolic stability, transport property and protein/tissue affinity) properties, which in turn ultimately determine molecule's pharmacokinetics (bioavailability and half-life), toxicity and pharmacodynamics (receptor affinity and efficacy). Poor absorption or permeation of a ligand is more likely when there are $\mathrm{MW}>500, \log \mathrm{Po} / \mathrm{w}>5$, number of $\mathrm{HBA}>10$, number of $\mathrm{HBD}>5$ and number of Rot $\mathrm{B}>5$. Hydrophobicity, membrane permeability and bioavailability are dependent on molecule's MW, Log P, Log S and HBA and HBD count. Molecules violating more than one of these rules may not exhibit optimum bioavailability. Sufficient water solubility is important for optimal bioavailability of drugs. Number of rotatable bonds is also important for molecular conformational studies (i.e., stereoselectivity of drug molecules) required for optimal binding with the receptor. Further, molecular PSA and MV are useful parameters for drug's transport properties ${ }^{[22-25]}$.

The results of predicted DL properties of the synthesized compounds, 5a-f is depicted in Table 3. All the compounds possess good drug-like properties based on Lipinski's rule of five with additional parameters such as Log S, PSA and MV. The values of the calculated drug-like properties are in acceptable range for all the compounds except $5 \mathrm{c}$ and $5 \mathrm{~d}$. These two compounds violated rule of five, $5 \mathrm{c}$ for having Log P-value 5.23 ( $>5$ ) and 5d for MW 519.99 (>500) and Log P 6.28 $(>5)$. Compounds $5 \mathrm{a}-\mathrm{f}$ possess less than or equal to 3 HBDs and have no more than 5 HBAs. The number of rotatable bonds, molecular PSA and MV values are in permissible range for all of the compounds. Furthermore, the drug-likeness score of the compounds, $5 \mathrm{a}-5 \mathrm{f}$ ranges from 0.07 to 0.78 . Compound $5 \mathrm{~d}$ exhibited highest drug-likeness score in the series though it violated two rules out of five. The results of druglikeness studies strongly suggest that newly designed hybrids have drug-likeness behaviour favourable for membrane permeability, transport and bioavailability and further interaction with the receptor. Assessment of drug-likeness score implies the suitability of hybrid derivatives as drug-like molecules. Compounds having zero or negative value are generally not considered as drug-like molecule.

Structure/property activity relationship of all newly designed compounds showed favourable DL properties; a reasonable correlation can be drawn between their calculated DL properties and in vitro antimalarial activity profile. $\log \mathrm{P}_{\mathrm{o} / \mathrm{w}}$ is a direct indicator of lipophilicity of

TABLE 3: PREDICTED DRUG-IIKENESS PROPERTIES

\begin{tabular}{|c|c|c|c|c|c|c|c|c|c|c|}
\hline $\begin{array}{l}\text { Compound } \\
\text { code }\end{array}$ & Mol. wt. & $\log P^{a}$ & $\log ^{b}$ & $\mathrm{HBA}^{\mathrm{c}}$ & $\mathrm{HBD}^{\mathrm{d}}$ & $\begin{array}{l}\text { Rot. } \\
\text { bonde }\end{array}$ & $\operatorname{PSA}^{f}\left(A^{2}\right)$ & $\begin{array}{l}M V^{g} \\
\left(A^{3}\right)\end{array}$ & $\begin{array}{l}\text { Rule } \\
\text { of } 5\end{array}$ & $\mathrm{DL}^{\mathrm{h}}$ \\
\hline $5 a$ & 393.83 & 3.53 & -6.36 & 4 & 3 & 5 & 70.21 & 328.79 & 0 & 0.47 \\
\hline $5 b$ & 407.86 & 3.81 & -6.39 & 4 & 3 & 6 & 70.21 & 346.59 & 0 & 0.44 \\
\hline $5 c$ & 441.87 & 5.23 & -7.93 & 4 & 3 & 4 & 66.22 & 367.60 & 1 & 0.44 \\
\hline $5 d$ & 519.99 & 6.28 & -10.10 & 4 & 3 & 6 & 69.61 & 445.22 & 2 & 0.78 \\
\hline $5 e$ & 419.87 & 3.88 & -7.62 & 4 & 1 & 2 & 56.62 & 353.32 & 0 & 0.07 \\
\hline $5 f$ & 442.08 & 4.86 & -8.34 & 5 & 3 & 4 & 77.36 & 404.26 & 0 & 0.47 \\
\hline
\end{tabular}

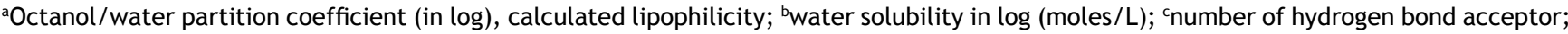
dnumber of hydrogen bond donor; fpolar surface area; ${ }^{\text {Im }}$ olar volume; ${ }^{\text {hdrug-likeness score }}$ 
drug substances. Higher the value of $\log$ P, better the biological membrane permeability. It is inevitable for a molecule to have sufficient lipophilicity which is required for its optimal bioavailability and biological action. HBA and donor groups are of paramount importance required to achieve optimal drug action. PSA is also closely related to the hydrogen bonding potential of a drug molecule. Practically, a compound with all drug-like properties in the desired range appears to exhibit high levels of therapeutic potency. Good DL properties and activity are complementary, and hence balanced attention to both properties and bioactivity could suitably transform a ligand to a good drug lead ${ }^{[25]}$. Better antimalarial activity of the compound $5 \mathrm{a}$ is probably due to the increased lipophilicity of the molecule. Polar properties such as HBD and HBA groups, rotatable bonds and PSA which contribute transport property to the drug molecule are all well balanced with the lipophilicity. Because of having desired lipophilicity (Log P 3.53 ) compound readily penetrated parasite's cell membrane that led to attain an intracellular concentration which was favourable for antimalarial action. DL score for this compound was 0.47 , which is within acceptable range. From above considerations, it can be attributed that 7-chloro4-aminoquinoline moiety coupled with 3-amino-2hydroxy-1,4-naphthoquinone system has potential as a new hybrid antimalarial scaffold. Different diamino functions of alkyl/aryl/heteroaryl groups present as connecting/bridge moiety may have contributing effects towards improving the antimalarial efficacy of parent hybrid skeleton. Some degree of variations in activity among synthesized hybrid compounds might be because of a small chemical diversity seen in the bridging pattern in different structural analogues. Small alkyl group (diaminoethyl, 5a) present as bridge moiety appears to be optimal structural requirement for the antimalarial activity. Increase in the length of carbon chain from 2-3 (diaminoethyl, 5a to diaminopropyl, 5b) the activity declines. Compounds with bulky aromatic bridge moieties such as 2,3-diaminophenyl (5c) and diamino-1-naphthylethyl (5d) are considerably less important for the activity. But, in case of mixed alkyl-aryl function the activity is retained as seen with the latter case. The heteroaryl groups found in compounds 5e (1,4-piperidyl) and $5 \mathrm{f}$ (2,6-diaminopyridyl) are almost equipotent to each, but were comparatively less potent than compounds with alkyl bridge moieties, particularly 5a. This implies that heteroaromatic groups as bridge moiety contribute to a limited extent to the overall antimalarial activity of a designed hybrid molecule unlike alkyl groups. The antimalarial potency of our present hybrid analogues is consistent with reports from earlier studies that CQchalcone acts against malaria parasites targeting haem polymerization in the digestive vacuole of parasites with possible resistant preventing action ${ }^{[15]}$. In addition, 1,4-naphthoquinone system mediated redox reactions/ alkylation mechanism in the parasite (involving two carbonyl sites) is attributed to be the secondary mode of action of antimalarial action for newly designed quinoline-lawsone hybrids ${ }^{[10]}$.

The present work reports the synthesis, in vitro antimalarial activity evaluation and drug-likeness prediction of some new quinoline-lawsone hybrids. The synthesis and antimalarial activity of newly designed hybrid molecules have not been reported earlier. All the synthesized hybrid compounds exhibited some degree of in vitro antimalarial activity against both CQ-sensitive and resistant strains of $P$. falciparum at the tested dose, which, however, was considerably less than that of the standard drug, CQ. Among synthesized compounds, compound with diaminoethyl conjugating moiety possesses better activity profile against both sensitive and resistant strains than rest of the synthesized analogues. Because of having favourable druglikeness behaviour a reasonable correlation appears to exist between drug-like properties of quinolinelawsone hybrids and their in vitro antimalarial activity. Newly reported quinoline-lawsone hybrids therefore have potential to be used as structural leads in the development of potent antimalarial drugs for the treatment of resistant malaria.

\section{Acknowledgements:}

The authors are thankful to SAIF, Punjab University, Chandigarh (India) for providing the spectral data of synthesized compounds.

\section{Conflicts of interest:}

There are no conflicts of interest.

\section{Financial support and sponsorship:}

Nil.

\section{REFERENCES}

1. WHO. World Malaria Report. Geneva: WHO;2015.

2. Rudrapal M, Chetia D, Prakash A. Synthesis, antimalarial and antibacterial activity evaluation of some new 4-aminoquonoline derivatives. Med Chem Res 2013;22:3703-11. 
3. Sashidhara KV, Kumar K, Dodda RP, Krishna NN, Agarwal P, Srivastava K, et al. Coumarin-trioxane hybrids: Synthesis and evaluation as a new class of antimalarial scaffolds. Bio org Med Chem 2012;22:3926-30.

4. Roy S, Chetia D, Rudrapal M, Prakash A. Synthesis and antimalarial activity study of some new Mannich bases of 7-chloro-4-aminoquinoline. Med Chem 2013;9:379-83.

5. Pandey AV, Bisht H, Babbarwal VK, Srivastava J, Pandey $\mathrm{KC}$, Chauhan VS. Mechanism of malarial haem detoxification inhibition by chloroquine. Biochem J 2001;355:333-8.

6. Dinio T, Gorka AP, McGinniss A, Roepe PD, Morgan JB. Investigating the activity of quinine analogues versus chloroquine resistant Plasmodium falciparum. Bio Org Med Chem 2012;20:3292-7.

7. Kyle DE, Teja-Isavadharm P, Li Q, Leo K. Pharmacokinetics and pharmacodynamics of qinghaosu derivatives: How do they impact on the choice of drug and the dosage regimens? Med Trop 1998;58:38-44.

8. Meshnick SR. Artemisinin: mechanisms of action, resistance and toxicity. Int J Parasitol 2002;32:1655-60.

9. de Rezende LC, Fumagalli F, Bortolin MS, de Oliveira MG, de Paula MH, de Andrade-Neto VF, et al. In vivo antimalarial activity of novel 2-hydroxy-3-anilino-1,4-naphthoquinones obtained by epoxide ring opening reaction. Bioorg Med Chem Lett 2013;23:4583-6.

10. Pingaew R, Prachayasittikul V, Worachartcheewan A, Nantasenamat C, Prachayasittikul S, Ruchirawat S, et al. Novel 1,4-naphthoquinone-based sulfonamides: Synthesis, QSAR, anticancer and antimalarial studies. Eur J Med Chem 2015;103:446-59.

11. Sharma D, Chetia D, Rudrapal M. Design, Synthesis and Antimalarial Activity of Some New 2-hydroxy-1,4naphthoquinone-4-hydroxyaniline Hybrid Mannich bases. Asian J Chem 2016;28:782-8.

12. Chadwick J, Amewu RK, Marti F, Garah FB, Sharma R, Berry $\mathrm{NG}$, et al. Antimalarial mannoxanes: hybrid antimalarial drugs with outstanding oral activity profiles and a potential dual mechanism of action. Chem Med Chem 2011;6:1357-61.

13. Muregi FW, Ishih A. Next-generation antimalarial drugs: Hybrid molecules as a new strategy in drug design. Drug Dev Res 2010;71:20-32.

14. Walsh JJ, Bell A. Hybrid drugs for malaria. Curr Pharm Des 2009;15:2970-85.
15. Sashidhara KV, Avula SR, Palnati GR, Singh SV, Srivastava $\mathrm{K}$, Puri SK, et al. Synthesis and in vitro evaluation of new chloroquine-chalcone hybrids against chloroquine-resistant strain of Plasmodium falciparum. Bioorg Med Chem 2012;22:5455-9.

16. Manohar S, Rajesh UC, Khan SI, Tekwani BL, Rawat DS. Novel 4-aminoquinoline-pyrimidine based hybrids with improved in vitro and in vivo antimalarial activity. ACS Med Chem Lett 2012;3:555-9.

17. O’Neil PM, Mukhtar A, Stocks PA, Randle LE, Hindley S, Ward SA, et al. Isoquine and related amodiaquine analogues: a new generation of improved 4-aminoquinoline antimalarials. J Med Chem 2003;46:4933-45.

18. Kumawat MK, Chetia D. Synthesis, antimalarial activity evaluation and molecular docking studies of some novel dispiro-1,2,4,5-tetraoxanes. Bangladesh J Pharmacol 2015;10:917-23.

19. Trager W, Jensen JB. Human malaria parasites in continuous culture. Science 1976;193:673-5.

20. Lambros C, Vanderberg JP. Synchronization of Plasmodium falciparum erythrocytic stages in culture. J Parasitol 1976;65:418-20.

21. Silverstein RM, Webster FX, editors. Spectrometric identification of organic compounds. New York: John Wiley and Sons Ltd; 1963.

22. Lipinski CA, Lombardo F, Dominy BW, Feeney PJ. Experimental and computational approaches to estimate solubility and permeability in drug discovery and development settings. Adv Drug Deliv Rev 1997;23:3-26.

23. Umarani N, Ilango K, Valentina P, Ishwarya T. Molecular properties prediction, synthesis and bio-evaluation of triazines glued benzothiazole congeners. Indian J Pharm Edu Res 2012;46:366-71.

24. Hassan MF, Manal MA, Khalid AA, Khalid AK. Synthesis and biological evaluation of fluoropyrazolesulfonylurea and thiourea derivatives as possible antidiabetic agents. J Enz Inhib Med Chem. In Press 2016.

25. Kerns EH, Di L, editors. Drug-like properties: concepts, structure design and methods from adme to toxicity optimization. New York: Academic Press; 2008. 